\title{
Auricular Acupuncture Versus Progressive Muscle Relaxation in Patients with Anxiety Disorders or Major Depressive Disorder: A Prospective Parallel Group Clinical Trial
}

\author{
Lukas de Lorent ${ }^{1, *}$, Agorastos Agorastos ${ }^{1}$, Alexander Yassouridis ${ }^{2}$, \\ Michael Kellner ${ }^{1}$, Christoph Muhtz ${ }^{1,3}$
}

${ }^{1}$ Department of Psychiatry and Psychotherapy, University Medical Center HamburgEppendorf, Hamburg, Germany

${ }^{2}$ Max Planck Institute of Psychiatry, Munich, Germany

${ }^{3}$ Department of Psychosomatic Medicine and Psychotherapy, University Medical Center

Hamburg-Eppendorf \& Schön Klinik Hamburg-Eilbek, Hamburg, Germany

Available online 22 April 2016

Received: Oct 13, 2015

Revised: Mar 1, 2016

Accepted: Mar 3, 2016

\section{KEYWORDS}

acupuncture;

anxiety;

auricular acupuncture;

depression;

NADA;

progressive muscle

relaxation

\begin{abstract}
Although acupuncture treatment is increasingly in demand among psychiatric patients, to date no studies have investigated the effectiveness of auricular acupuncture (AA) in treating anxiety disorders or major depressive disorder. Thus, this study aimed to compare the effectiveness of AA versus progressive muscle relaxation (PMR), a standardized and accepted relaxation method. We examined 162 patients with a primary diagnosis of anxiety disorder or major depressive disorder, and each patient chose between treatment with AA, executed according to the National Acupuncture Detoxification Association protocol, and treatment with PMR. Each group had treatments twice a week for 4 weeks. Before and after treatment, each participant rated four items on a visual analog scale: anxiety, tension, anger/aggression, and mood. Statistical analyses were performed with the original visual analog scale scores and the Change-Intensity Index, an appropriate indicator of the difference between two values of a variable. Our results show that
\end{abstract}

This is an Open Access article distributed under the terms of the Creative Commons Attribution Non-Commercial License (http:// creativecommons.org/licenses/by-nc/4.0) which permits unrestricted non-commercial use, distribution, and reproduction in any medium, provided the original work is properly cited.

* Corresponding author. University Medical Center Hamburg-Eppendorf, Department of Psychiatry and Psychotherapy, Martinistrasse 52, Hamburg 20251, Germany.

E-mail: lukasdelorent@googlemail.com (L. de Lorent). 
treatment with AA significantly decreased tension, anxiety, and anger/aggression throughout the 4 weeks, but did not elevate mood. Between AA and PMR, no statistically significant differences were found at any time. Thus, we suggest that both AA and PMR may be useful, equally-effective additional interventions in the treatment of the above-mentioned disorders.

\section{Introduction}

Acupuncture treatment is increasingly in demand among psychiatric patients and many people report subjectively helpful effects. In many cases, current pharmacological treatment of anxiety disorders (AD) [1-3] and major depressive disorder (MDD) [4-6] is not able to achieve remission. Eisendrath et al [6] report on up to $60 \%$ of MDD patients not being fully cured after 1 year of treatment. Thus, it appears necessary to give more attention to complementary treatments. The effectiveness of acupuncture in the treatment of $A D$ [7-9] and MDD [10-12] remains unclear, owing to the strong distinctions of study results, the high diversity in methodological study design, and varying inclusion of studies in review research [13]. Further trials using standardized methods are required [14].

In this study, acupuncture was executed according to the National Acupuncture Detoxification Association (NADA) protocol: a standardized and comparable method of auricular acupuncture (AA) with ease of application and limited adverse reactions [15], which seems to have high acceptance among psychiatric patients [16]. This method was introduced as an additional relaxation technique on our unit, and includes the needling of five specific acupuncture points on both ears [17]. It was originally developed for patients withdrawing from substance abuse and has been the primary element of the New York Lincoln Hospital detoxification program, where it was established in 1985 [18]. Today, several studies suggest the use of AA in withdrawal treatment [19-21], although recent research reviews could not confirm the effectiveness of $A A$ [22] or even the NADA protocol [15] in substance abuse treatment.

As a comparative relaxation treatment, progressive muscle relaxation (PMR) was applied in our study. PMR has been well established for many decades, especially for the treatment of severe states of physical and mental tension, which is a common symptom of anxiety disorders [23-26] and depression [27,28]. Many studies also suggest its potential utility in the treatment program of many other illnesses which are associated with conditions of anxiety and depression, such as schizophrenia $[29,30]$, sleeping disorders [31], endometriosis [32], atopic dermatitis [33], and cancer [34,35].

The main aim of this study was to examine the effectiveness of AA according to the NADA protocol versus PMR in patients with $A D$ or MDD. We hypothesized that both treatments show significant effects in alleviating the examined items "tension," "anxiety," "anger/aggression," and "state of mood," and that there is no significant difference between these two treatments. In order to increase the patients' treatment acceptance and compliance, this study was set up in an open design in which each patient was freely able to choose between both treatments. We additionally presumed that, due to the more passive participation in AA therapy rather than PMR, patients choosing treatment with $\mathrm{AA}$ would show a higher rate of regressive tendencies.

\section{Materials and methods}

\subsection{Participants}

We examined 162 patients with a primary diagnosis of AD or MDD. All patients were treated on the psychiatric ward or day hospital of the anxiety spectrum disorders unit at the Department of Psychiatry and Psychotherapy, University Medical Center Hamburg Eppendorf, Germany. All participants provided written informed consent before participating in the study, which was conducted in accordance with the Declaration of Helsinki and approved by the ethics committee of the Medical Board of Hamburg.

All volunteers met the Diagnostic and Statistical Manual of Mental Disorders IV (DSM-IV) diagnostic criteria for the above-mentioned disorders according to a Structured Clinical Interview for DSM-I interview [36], which was administered by two board-certified psychiatrists. These psychiatrists were working independently and were not included in either the acupuncture or PMR treatment. Furthermore, we gathered data on additional parameters such as age, sex, number of secondary psychiatric diagnoses, and current medication (see Table 1).

In order to rate the patients' regressive tendencies (signs of dependence), we used the FAPK (questionnaire for the assessment of psychosomatic disorder process), subscale eight [37].

All patients underwent physical and complementary laboratory-chemical examination of blood and urine. Participants with ear infections, anamnestic substance abuse, or positive drug screening, as well as patients taking opioid analgesics and patients suffering from schizophrenic or organic cerebral psychosis, were excluded.

All patients received a multimodal treatment including a personal and group cognitive behavior therapy setting and psychopharmacological treatment.

\subsection{Procedures}

After standardized oral and written presentation of each procedure, each participant chose voluntarily between AA treatment and PMR treatment in a group setting. The 
Table 1 Demographics and clinical characteristics.

\begin{tabular}{|c|c|c|c|}
\hline \multirow[t]{2}{*}{ Characteristics } & \multirow{2}{*}{$\begin{array}{l}\text { AA group } \\
(n=90)\end{array}$} & \multirow{2}{*}{$\begin{array}{c}\text { PMR group } \\
(n=72)\end{array}$} & \multirow[t]{2}{*}{$p^{*}$} \\
\hline & & & \\
\hline \multicolumn{4}{|l|}{ Distribution of disorders } \\
\hline$A D$ & $53(58.9 \%)$ & $43(59.7 \%)$ & 0.4573 \\
\hline MDD & $37(41.1 \%)$ & $29(40.3 \%)$ & 0.4573 \\
\hline Study participation & $84(93.3 \%)$ & $52(72.2 \%)$ & $0.0001^{\dagger}$ \\
\hline Drop out & $30(33.3 \%)$ & $28(38.9 \%)$ & 0.2317 \\
\hline \multicolumn{4}{|l|}{ Sex } \\
\hline Men & $61(67.8 \%)$ & $42(58.3 \%)$ & 0.1073 \\
\hline Women & $29(32.2 \%)$ & $30(41.7 \%)$ & 0.1073 \\
\hline Age & 34.1 (SEM \pm 1.139$)$ & $34.7(\mathrm{SEM} \pm 1.351)$ & 0.945 \\
\hline Patients with sec. psych. diagnoses & $56(62.2 \%)$ & $46(63.9 \%)$ & 0.4137 \\
\hline \multicolumn{4}{|l|}{ Medication } \\
\hline Antidepressants & $80(88.9 \%)$ & 59 (81.9\%) & 0.1041 \\
\hline Antipsychotics & $37(41.1 \%)$ & $19(26.4 \%)$ & 0.0251 \\
\hline Benzodiazepines prn & $3(3.3 \%)$ & $2(2.8 \%)$ & 0.0419 \\
\hline Nonbenzodiazepines & $23(25.6 \%)$ & $9(12.5 \%)$ & 0.0190 \\
\hline Mood stabilizers & $10(11.1 \%)$ & $3(4.2 \%)$ & 0.0529 \\
\hline
\end{tabular}

setting involved not more than eight patients at a time and was performed in a sitting position. All participants started and finished treatment at the same time. Each session lasted 30 minutes and took place twice a week over a period of 4 weeks. Both types of treatment were provided by skilled medical personnel. Shortly before and immediately after group treatment, each participant rated four different items on a $0-100-\mathrm{mm}$ visual analog scale (VAS): "tension," "anxiety," "anger/aggression" (VAS $0=$ none, 100 = most imaginable), and "state of mood" (VAS $0=$ bad, $100=$ good). The VAS was handed out by the group therapist and was returned anonymously by the participants. Before the first treatment, each patient was introduced to the use of the VAS by the psychiatrist. For inclusion into study analyses, participation of at least 1 week was necessary.

\section{3. $\mathrm{AA}$}

In this study, AA was executed according to the NADA protocol. It included the needling of five specific acupuncture points on both ears: point 51 (Sympathetic point), point 55 (Shen Men), point 95 (Kidney point), point 97 (Liver point), and point 101 (Lung point) [17]. All acupuncturists were nurses, specially trained, and certified in theory and practice of the use of the NADA protocol by Ralph Raben, MD, chairman of NADA Germany. They had at least 2 years' experience in application of the NADA protocol. All points were acupunctured with fine sterile single-use steel needles, sized $0.25 \times 20 \mathrm{~mm}$. The needles were inserted about 2-3 mm deep, so that the needle point pierced the skin and was positioned in the cartilage of the ear. They remained in position for 30 minutes. To increase transparency and comparability, we used the Standards for Reporting Interventions in Clinical Trials of Acupuncture [38] in this study.

\subsection{PMR}

Patients who chose treatment with PMR were treated in a group setting, carried out by the method of Bernstein and Borkovec [39]. All participants were asked to close their eyes while sitting comfortably. Each group session included 16 main exercises of consciously tensing and relaxing specific groups of muscles.

\subsection{Statistical analysis}

Since patients attended treatment twice a week, we first calculated averaged values of the two pre- and post-VAS scores to receive one pretreatment-, one post-treatment-, and the resulting delta-VAS score for each patient and each week. These averaged values were used for further statistical analyses.

Due to incomplete data in the present study, an intention-to-treat (ITT) analysis seemed to be adequate. However, instead of ITT we decided to use another concept by defining an appropriate indicator, called Change-Intensity $(\mathrm{Cl})$ Index. This index is an analog to the "activation intensity" quantity defined by A. Yassouridis [40]. Based on the $\mathrm{Cl}$-Index we were able to avoid the difficulty of dropouts in a more convincing and reliable manner than with ITT and last observation carried forward.

The $\mathrm{Cl}-$ Index is defined as follows: 
$C l=\left\{\begin{array}{cc}200 x \frac{\left(Y_{B}^{2}+0.000005\right)}{\left(Y_{B}^{2}+Y_{A}^{2}+0.00001\right)}(i n \%), & \text { if } Y_{A} \text { and } Y_{B} \text { are both not missing. } \\ 100(\text { in } \%), & \text { if } Y_{A} \text { or } Y_{B} \text { is missing. }\end{array}\right.$

where $Y_{B}$ and $Y_{A}$ are values of an examined variable under condition $A$ (no treatment) or condition $B$ (treatment with $A A$ or $P M R$ ). If $Y_{B}$ and $Y_{A}$ are equal, $C l$ is 100 (\%). In this case, the treatment applied was not effective. The more the $\mathrm{Cl}$ Index diverges from $100 \%$ towards $200 \%$ or $0 \%$, the stronger or weaker $Y_{B}$ differs from $Y_{A}$, which demonstrates the treatment's effectiveness. From the definition formula it can be deduced that the $\mathrm{Cl}$-Index is robust against outliers, produces data homogeneity, and since missing values of the variables are per definition substituted by values denoting standoff situation, it does not impair the statistical power of the study, irrespective of the number of dropouts.

To prove significant ad hoc therapy effects of AA and PMR across time, that is, post-treatment versus pretreatment changes in VAS scores of the considered items in each week, we first calculated corresponding $\mathrm{Cl}$-Indices for the averaged VAS scores in each week. On the obtained $\mathrm{Cl}$-Indices, one-sample $t$-tests were applied in order to test the null hypothesis (pre- and post-treatment VAS scores do not differ significantly) for each week and each treatment.

The $\mathrm{Cl}$-Indices were further used to compare the two treatments in their therapy effects over time. For this comparison, analyses of covariance using treatment as a between-subjects factor, time as a within-subjects factor, and sex and age as covariates were performed.
In case of significant factor effects in the analyses of covariance, univariate F-tests or tests with contrasts followed to localize simple effects.

Differences between AA and PMR in patients' regressive tendencies and the number of secondary psychiatric diagnoses were tested for significance using the Man$\mathrm{n}$-Whitney $\mathrm{U}$ tests, whereas differences in age, sex, and primary diagnosis within the two treatment groups were tested using $\chi^{2}$ tests.

As nominal level of significance an alpha $=0.05$ was accepted. However, posthoc tests (univariate F-tests or tests with contrasts) were performed with an adjusted alpha (according to the Bonferroni procedure) in order to keep the type I error $<0.05$. If possible, values in tables and figures are given as mean \pm standard error of the mean.

\section{Results}

\subsection{Participants}

Among all 162 participants, there were 96 with the primary diagnosis of $A D$ [including obsessive-compulsive disorder $(n=41)$, agoraphobia with or without panic disorder $(n=22)$, post-traumatic stress disorder $(n=18)$, phobias $(n=8)$, generalized anxiety disorder $(n=4)$, and panic

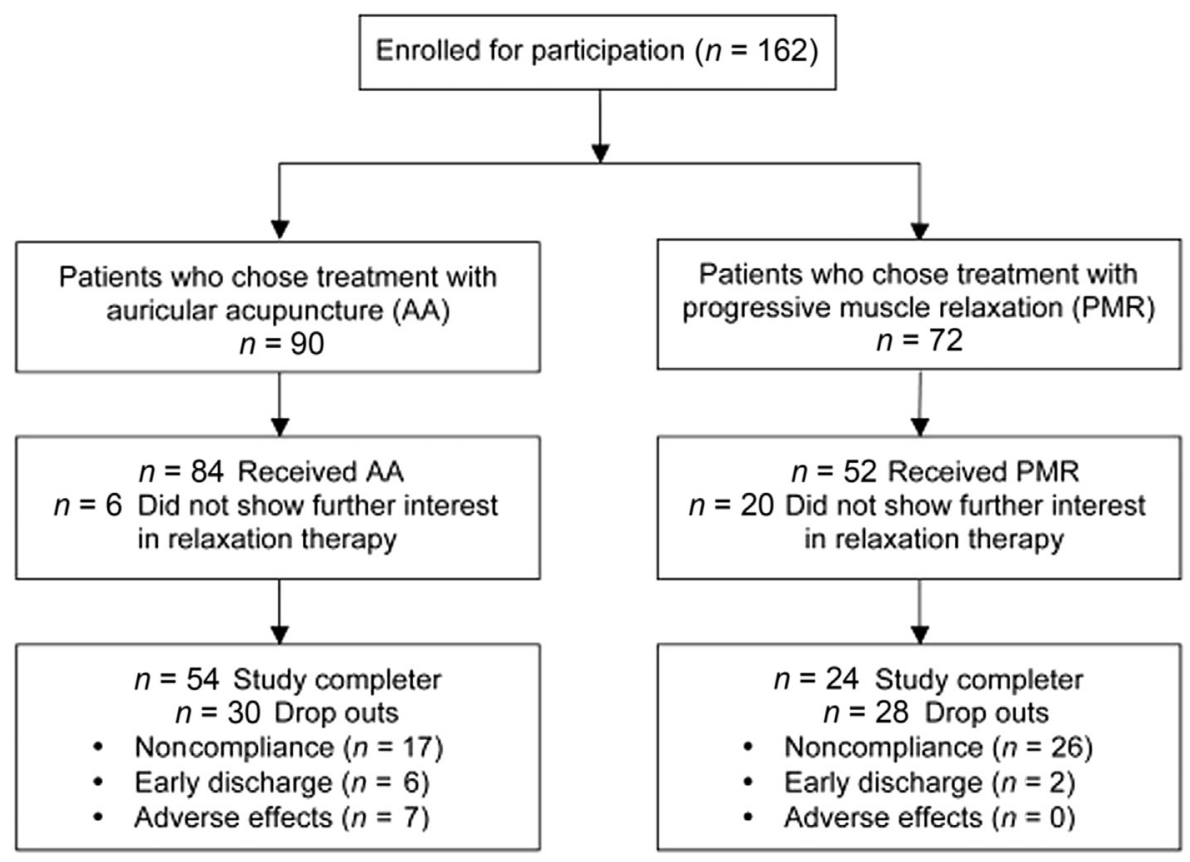

Figure 1 Flow of participants. 
disorder $(n=3)$ ] and 66 with MDD (Table 1). Fig. 1 gives an overview of participation and dropouts in this study: 90 patients chose treatment with $A A$ and 72 chose treatment with PMR. Eighty-four patients from the AA group and 52 patients from the PMR group actually participated in the study, while the rest ( $n=6$ from the AA group, $n=20$ from the PMR group) did not show further interest in relaxation therapy. Throughout the study, we had a dropout rate of $35.8 \%$ ( $n=30$ from the AA group, $n=28$ from the PMR group). Reasons for this were noncompliance $(n=43)$, early discharge $(n=8)$, or adverse effects $(n=7$ within the AA group) such as headache $(n=2)$, local pain $(n=4)$, or circulatory problems $(n=1)$. Of all the patients, $28.39 \%$ were without psychopharmacological treatment, while the rest were treated with either one (29.01\%), two (30.25\%), three $(9.88 \%)$, or more than three $(2.47 \%)$ types of psychotropic medication. Disorder distribution, sex, age, number of patients with secondary psychiatric diagnoses, and current medication did not differ between groups (Table 1). Although some medications indicate significant differences in their frequencies between treatment groups, at a Bonferroni-corrected level of significance they do not confirm it.

\subsection{Outcomes of VAS scores}

For both AA and PMR, VAS scores show improving results in all measured items (Figs. 2A-D). The greatest effects were seen on tension (Fig. 2B), whereas mood showed the lowest effects (Fig. 2D).

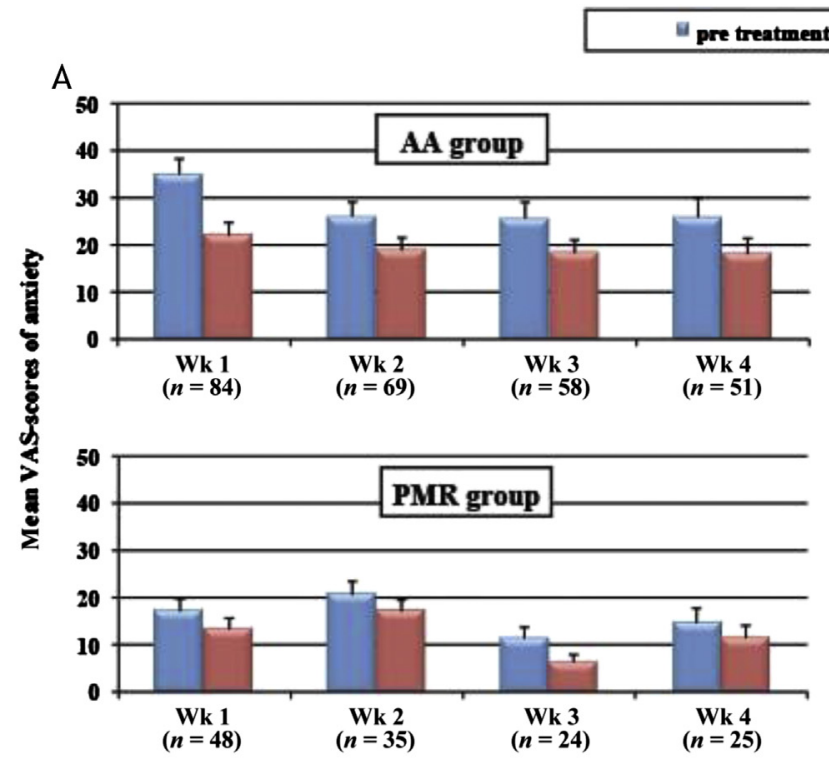

C
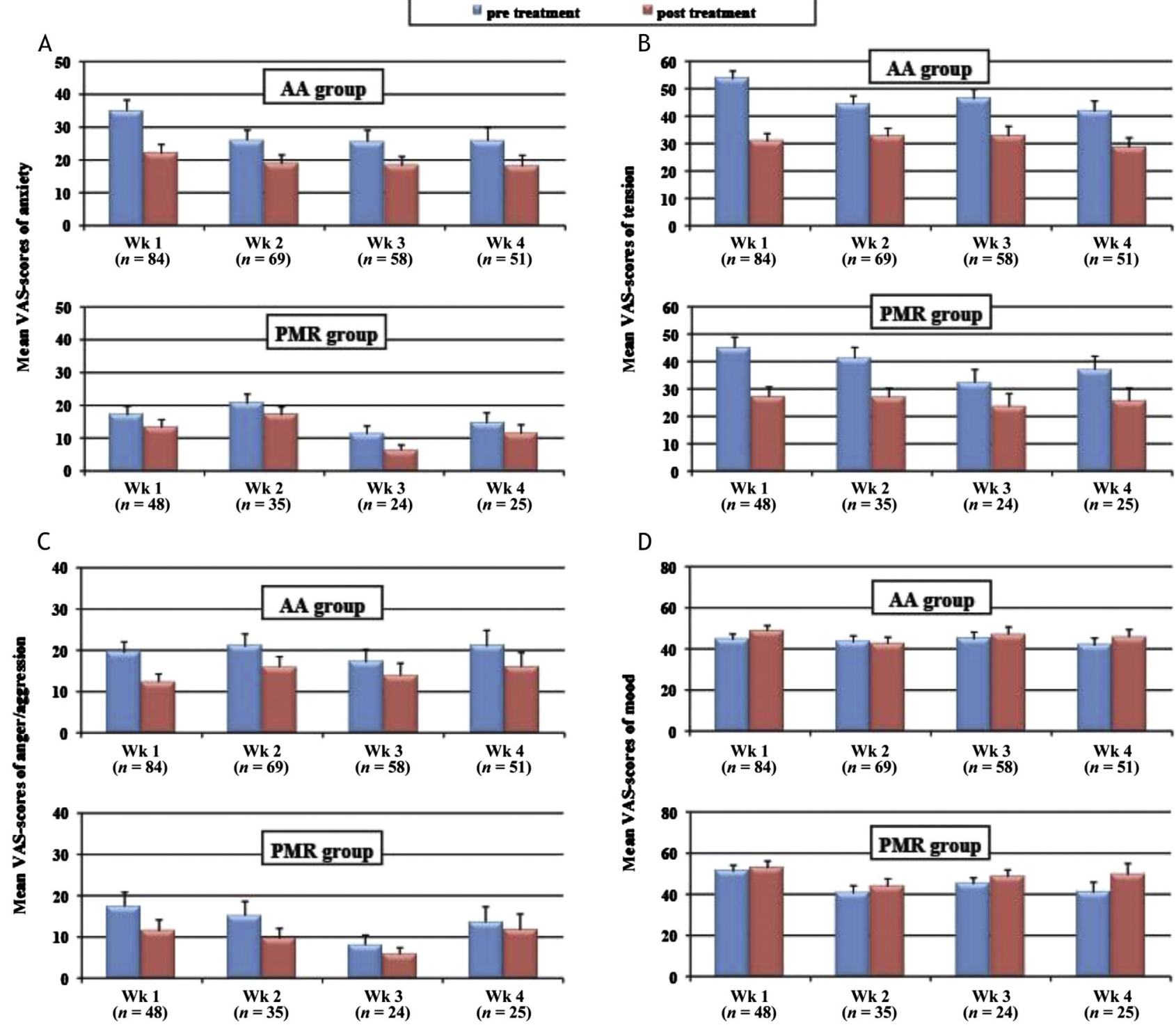

D

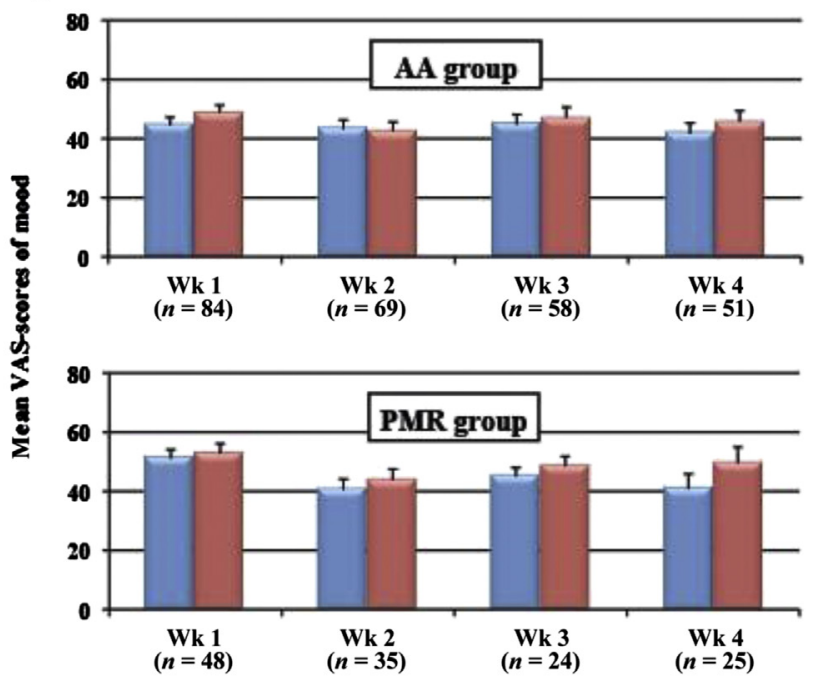

Figure 2 Means \pm standard error of the mean of the outcome parameters [visual analog scale (VAS) scores] of anxiety, tension, anger/aggression, and mood within each treatment group at single weeks. VAS are rated as follows: (A) "anxiety," (B) "tension," (C) "anger/aggression": VAS $0=$ none, $100=$ most imaginable; (D) "mood": VAS $0=$ bad, $100=$ good. AA = auricular acupuncture; PMR = progressive muscle relaxation. 
Table 2 Change-Intensity Indices of anxiety, tension, anger/aggression, and mood.

\begin{tabular}{|c|c|c|c|c|}
\hline \multirow[t]{2}{*}{ Symptoms by wk } & \multicolumn{2}{|l|}{ AA group } & \multicolumn{2}{|l|}{ PMR group } \\
\hline & Mean & $p$ & Mean \pm SEM & $p$ \\
\hline \multicolumn{5}{|l|}{ Anxiety } \\
\hline Wk $1(n=136)$ & $68.44 \pm 5.92$ & 0.000 & $76.78 \pm 5.6$ & .000 \\
\hline Wk $2(n=104)$ & $81.20 \pm 5.13 c$ & 0.000 & $92.27 \pm 4.8$ & 0.118 \\
\hline $7=82)$ & $85.25 \pm 5.03 c$ & 0.004 & $84.58 \pm 4.7$ & .002 \\
\hline Wk 4 ( & 89.53 & 0.017 & $95.41 \pm$ & כונים \\
\hline \multicolumn{5}{|l|}{ Tension } \\
\hline$=136)$ & $55.16 \pm 4.91 c$ & 0.000 & $57.23 \pm 6.03$ & .000 \\
\hline$=104)$ & $74.58 \pm 4.83 c$ & 0.000 & $74.31 \pm 5.3$ & .000 \\
\hline Wk & $77.36 \pm 4.63 c$ & 0.000 & $86.27 \pm 4.14$ & 002 \\
\hline Wk $4(n=76)$ & $81.36 \pm 4.52 c$ & 0.000 & $82.74 \pm 5.00$ & .001 \\
\hline \multicolumn{5}{|l|}{ Anger/aggression } \\
\hline Wk $1(n=136)$ & $76.95 \pm 5.79 c$ & 0.000 & $74.70 \pm 5.95$ & .000 \\
\hline$=104)$ & $85.72 \pm 4.70 c$ & 0.003 & $80.36 \pm 5.6$ & .001 \\
\hline$=82)$ & $87.40 \pm 5.04 C$ & 0.014 & $93.74 \pm 5.95$ & 298 \\
\hline Wk $4(n=76)$ & $84.79 \pm 4.58$ & 0.001 & $86.17 \pm 5.41$ & ( \\
\hline \multicolumn{5}{|l|}{ Mood } \\
\hline Wk 1 & 112.85 & & $.19 \pm 4$. & 399 \\
\hline$=104)$ & 103.90 & & $104.77 \pm 4.4$ & 286 \\
\hline Wk $3(n=82)$ & $101.17 \pm 4.53 c$ & 7 & $102.83 \pm 3.05$ & 358 \\
\hline Wk $4(n=76)$ & $107.23 \pm 3.91 \mathrm{c}$ & & & 0.022 \\
\hline \multicolumn{5}{|c|}{$\begin{array}{l}\text { Means } \pm \text { standard error of the mean of the Change-Intensity } \\
\text { Index of the outcome parameters (delta visual analog scale } \\
\text { scores) of anxiety, tension, anger/aggression, and mood within } \\
\text { each treatment group at single weeks. The more the Change- } \\
\text { Intensity Index diverges from } 100 \% \text { towards } 200 \% \text { or } 0 \% \text {, the } \\
\text { stronger it demonstrates the treatment's effects compared to } \\
\text { baseline (no treatment). The } p \text { values indicate the significance } \\
\text { of the deviation of the Change-Intensity Index from } 100 \text { (no- } \\
\text { treatment effect) by one sample } t \text {-tests. No-treatment scores } \\
\text { are theoretically mathematically assumed reference scores. } \\
\text { AA = auricular acupuncture; PMR = progressive muscle relax- } \\
\text { ation; SEM = standard error of the mean. }\end{array}$} \\
\hline
\end{tabular}

\subsection{Ad hoc therapy effects of $A A$ and PMR}

As shown in Table 2, AA points to meaningful ad hoc effects on anxiety, tension, and anger/aggression in all 4 weeks. In each week, mean $\mathrm{Cl}$-values of these items were significantly less than 100 , indicating a significant improvement after treatment in each week (one-sample $t$-tests, $p<\alpha_{\mathrm{B}}$, where $\alpha_{B}$ Bonferroni corrected alpha $=0.05$ ). Treatment with PMR also points to meaningful effects on anxiety, tension, and anger/aggression, but not throughout all weeks. It appears to have had continuous effects only on the outcomes of tension $\left(p<\alpha_{B}\right.$, where $\alpha_{B}$ Bonferroni corrected alpha $=0.05$ ), whereas the effects on the outcomes of anxiety and anger/aggression did not show steadily improving results. The outcomes of mood rarely improved: The effects of AA were seen only within the $1^{\text {st }}$ week, and the effects of PMR only within the last week.

\subsection{Treatment comparison of ad hoc effects over time}

Multivariate analyses of covariance applied on the $\mathrm{Cl}$ Indices of the considered items revealed that there was a significant effect of time on anxiety and tension [multivariate tests of significance; anxiety $\rightarrow F(3.132)=4.79$, significance of $F=0.003$, power $=0.941$; tension $\rightarrow$ $F(3.132)=16.08$, significance of $F<0.0001$, power $=1.000$ ], but no significant effects of treatment or time-treatment interaction on these two outcome variables. The $\mathrm{Cl}$-Indices of anger/aggression and mood were not significantly affected either by time or by treatment, or by time-treatment interaction. There were no influences by the covariates sex and age. Between the AA and PMR groups, no significant distinctions could be found at any time.

Subsequent tests with contrasts in analysis of covariance across the time-factor levels pointed for both items (anxiety and tension) and both treatments (AA and PMR), with significantly higher values in the $1^{\text {st }}$ week than in the following weeks (tests with contrasts, $p<\alpha_{B}$, where $\alpha_{B}$ Bonferroni corrected alpha $=0.05$ ). Note that although the anxiety VAS scores at baseline (Fig. 2A) are statistically different between $\mathrm{AA}$ and PMR, the $\mathrm{Cl}$ of the VAS scores between pre- and post-treatment did not differ significantly between treatments.

An interesting question would be whether baseline values and eventual cross-sectional correlations of "anxiety," "tension," "state of mood," and "anger/aggression" in the intervention sessions may influence the discovered time effects. To answer this question, we additionally performed a multivariate analysis of covariance by simultaneously considering the $\mathrm{Cls}$ of the four variables as dependent variables; treatment and time as influential factors; and age, sex, and the baseline values of the considered variables as covariates. Besides a significant main time effect (Wilks multivariate tests of significance; effect of time: $F_{12,119}=5.21$, significance of $F<0.0001$, power $=1.000$ ) the other two effects, that is, the treatment and the interaction "treatment-time" effect were not significant. The time effect was significant on anxiety and tension (univariate $F$-tests in analyses of covariance, $p<\alpha_{B}$, where $\alpha_{B}$ Bonferroni corrected alpha $=0.05)$, a result identical to that reported above. The covariates now showed a significant effect (Wilks multivariate tests of significance; effect of the covariates: $F_{24,423}=3.27$, significance of $F<0.0001$, power $=1.000$ ) predominantly caused by a significant association of the anxiety at baseline to the $\mathrm{Cl}$ values of anxiety in the other intervention sessions. Since factors identified in covariance analyses are adjusted from covariate influences, we can assert that the findings regarding time effects on anxiety and tension are robust.

\subsection{Completer analyses}

Additional exploratory and confirmatory analyses including only completer patients, that is, patients who participated in treatment for the entire 4-week period, did not decisively differ from aforementioned study results (not significant).

\subsection{Patients' regressive tendencies}

Fifty-eight patients from the AA group and 40 patients from the PMR group filled out the FAPK questionnaire subscale 
eight (maximum attainable value: 23 ). No significant differences in regressive tendencies could be found between AA $(8.74 \pm 0.53)$ and PMR $(7.65 \pm 0.57)$ treatment choice (Mann-Whitney $U$ test, $p$-values not significant).

\section{Discussion}

This study was set up as a prospective parallel group clinical trial and investigated the effectiveness of $A A$ according to the NADA protocol versus PMR in patients with AD or MDD. It revealed, in agreement with our initial hypothesis, that there are no significant differences between these two treatments. Both treatments showed significant effects in reducing anxiety, tension, and anger/aggression, which affirms our patients' subjective assessment and justifies the practice of these two treatments. Furthermore, patients' regressive tendencies did not affect treatment choice.

To date, there are no studies investigating the effectiveness of $A A$ in the treatment of $A D$ or MDD. However, several investigations show preliminary evidence for the effectiveness of AA in reducing anxiety symptoms, for example, before dental treatment $[41,42]$ or other ambulatory surgical interventions $[43,44]$, or among healthy persons [45]. Wu et al [43] concluded that both body acupuncture and $\mathrm{AA}$ are effective in reducing anxiety before surgery, but due to its applicability, AA "seems to have more wide usage in future anxiety treatments." According to Acar et al [46] and Paraskeva et al [47], other facial points also seem to be appropriate for lowering preoperative anxiety. In 2011, Black et al [48] set up a trial investigating the anxiety-lowering effect of the NADA protocol among patients withdrawing from psychoactive drugs. However, in comparison to other control treatments including sham AA, it was not found to be more effective in reducing withdrawal-related anxiety.

It seems quite interesting that both $\mathrm{AA}$ and PMR were able to improve tension, anxiety, and anger/aggression, but not mood. This indicates that tension, anxiety, and anger/ aggression might be influenced more easily than mood by relaxation therapy. We can assume that mood is an emotional state, which is more stable and obviously, in the short term, not as easily improved as other conditions; this also might be a reason for the higher rate of dropouts in depressed patient collectives (see below) [49]. This interesting result deserves more attention in further studies.

This first trial examining the effectiveness of $A A$ in the treatment of $A D$ or MDD is encouraging. Unfortunately, our results cannot simply be compared with other studies using different forms of acupuncture, such as body acupuncture or electro-acupuncture. This basic problem of comparability within acupuncture studies has been described by many authors $[7,8,14]$.

Several limitations must be considered. Firstly, neither randomization nor blinding was applied. In order to increase the patients' treatment acceptance and compliance, this study was set up in an open design in which each patient was freely able to choose between both treatments. To determine whether treatment choices may have had an influence on study results, we gathered data on age, sex, secondary psychiatric diagnoses, and current medication. No differences were found, which implies that both treatment groups might have formed approximately equally. Due to our study design, it must also be considered that we were not able to eliminate possible placebo effects. Therefore, we suggest that sham AA should be included into future studies.

Secondly, since acupuncture and PMR were applied in a group setting, there might have been possible group effects, which were not further examined in this study. Investigating possible group effects in acupuncture treatment might be an important issue in future trials. Thirdly, our patient collective comprised $A D$ and MDD. While the summarization of highly comorbid disorders enabled us to set up a larger clinical real-world sample, it also means that our study results could not simply be transferred to each disorder individually. Fourthly, all patients received a multimodal treatment including psychopharmacological treatment. Thus, we were not able to exclude that medication also had an effect on study results, although there were no significant differences in medication distribution between both groups. Fifthly, the usage of VAS may have been afflicted with errors. The immediate assessment of personal mental conditions and, furthermore, the attempt to set pretreatment and post-treatment feelings into correct relation may have caused erroneous data. However, it can be assumed that within the multitude of data, most patients were able to make certain assessments. Sixthly, many patients did not participate in treatment throughout the total period of 4 weeks, which caused a decrease of sample size. A recent meta-analysis, investigating dropouts from cognitive behavioral therapy, also showed a high rate of $26.2 \%$ during treatment and even a higher rate of $36.4 \%$ for depression in subgroup analysis [49]. Interestingly, contrary to our expectations, free treatment choice did not decrease the dropout rate in this trial. Mischoulon et al [50], who also examined acupuncture treatment in MDD in an open design, reported about a similar dropout rate of $33 \%$. It seems that regardless of treatment choice, especially depressed patients show higher tendencies in discontinuing treatment. It can be assumed that this behavior is reducible to the main characteristics of this illness itself, such as listlessness, hopelessness, and lethargy.

This study is the first trial comparing the effectiveness of AA according to the NADA protocol and PMR among patients with $A D$ or MDD. Despite the limitations, this study suggests that both AA and PMR may be useful and equally effective additional interventions in the treatment of $A D$ and MDD. We suggest that randomization should be included into future study designs and that controlled studies using sham AA and blinding should be performed in this patient population.

\section{Disclosure statement}

The author declares to have no conflicts of interest and no financial interests related to the material of this manuscript.

\section{Acknowledgments}

We are grateful to the excellent work of Mrs Iris Wesser, Mrs Silvia Barth, and Mr Jens Bleier, who were responsible for 
the execution of the NADA protocol and the collection of the patients' VAS assessments.

\section{References}

[1] Pollack MH, Otto MW, Roy-Byrne PP, Coplan JD, Rothbaum BO, Simon NM, et al. Novel treatment approaches for refractory anxiety disorders. Depress Anxiety. 2008;25:467-476.

[2] Baldwin D, Woods R, Lawson R, Taylor D. Efficacy of drug treatments for generalised anxiety disorder: systematic review and meta-analysis. BMJ. 2011;342:d1199.

[3] Koen N, Stein DJ. Pharmacotherapy of anxiety disorders: a critical review. Dialogues Clin Neurosci. 2011;13:423-437.

[4] McIntyre RS, Filteau MJ, Martin L, Patry S, Carvalho A, Cha DS, et al. Treatment-resistant depression: Definitions, review of the evidence, and algorithmic approach. J Affect Disord. 2014;156:1-7.

[5] Berlim MT, Turecki G. Definition, assessment, and staging of treatment-resistant refractory major depression: a review of current concepts and methods. Can J Psychiatry. 2007;52: 46-54.

[6] Eisendrath SJ, Gillung E, Delucchi KL, Segal ZV, Nelson JC, Mclnnes LA, et al. A randomized controlled trial of mindfulness-based cognitive therapy for treatment-resistant depression. Psychother Psychosom. 2016;85:99-110.

[7] Errington-Evans N. Acupuncture for anxiety. CNS Neurosci Ther. 2012;18:277-284.

[8] Pilkington K. Anxiety, depression and acupuncture: a review of the clinical research. Auton Neurosci. 2010;157:91-95.

[9] Pilkington K, Kirkwood G, Rampes H, Cummings M, Richardson J. Acupuncture for anxiety and anxiety disorders-a systematic literature review. Acupunct Med. 2007;25: $1-10$.

[10] Smith CA, Hay PP, Macpherson H. Acupuncture for depression. Cochrane Database Syst Rev. 2010:CD004046.

[11] Wang H, Qi H, Wang BS, Cui YY, Zhu L, Rong ZX, et al. Is acupuncture beneficial in depression: a meta-analysis of 8 randomized controlled trials? J Affect Disord. 2008;111: $125-134$.

[12] Leo RJ, Ligot JS. A systematic review of randomized controlled trials of acupuncture in the treatment of depression. J Affect Disord. 2007;97:13-22.

[13] Ernst E, Lee MS, Choi TY. Acupuncture for depression?: a systematic review of systematic reviews. Eval Health Prof. 2011;34:403-412.

[14] Halbreich U. Systematic reviews of clinical trials of acupuncture as treatment for depression: how systematic and accurate are they? CNS Spectr. 2008;13:293-294, 299-300.

[15] D'Alberto A. Auricular acupuncture in the treatment of cocaine/crack abuse: a review of the efficacy, the use of the National Acupuncture Detoxification Association protocol, and the selection of sham points. J Altern Complement Med. 2004; 10:985-1000.

[16] Payer K, Ots T, Marktl G, Pfeifer F, Lehofer M. Patient satisfaction with the NADA-Protocol in a psychiatric ward - An observational study of practical application. Deutsche Zeitschrift fuer Akupuntur. 2007;50:10-13 [in German].

[17] Raben R. Acupuncture according to the NADA Protocol - an outline of treating addiction patients. Deutsche Zeitschrift fuer Akupuntur. 2004;47:35-40 [in German].

[18] Smith MO, Khan I. An acupuncture program for the treatment of drug-addicted persons. Bull Narc. 1988;40:35-41.

[19] Chen HH, Yeh ML, Chao YH. Comparing effects of auricular acupressure with and without an internet-assisted program on smoking cessation and self-efficacy of adolescents. J Altern Complement Med. 2006;12:147-152.
[20] Avants SK, Margolin A, Holford TR, Kosten TR. A randomized controlled trial of auricular acupuncture for cocaine dependence. Arch Intern Med. 2000;160:2305-2312.

[21] Shwartz M, Saitz R, Mulvey K, Brannigan P. The value of acupuncture detoxification programs in a substance abuse treatment system. J Subst Abuse Treat. 1999;17:305-312.

[22] Gates S, Smith LA, Foxcroft DR. Auricular acupuncture for cocaine dependence. Cochrane Database Syst Rev. 2006: CD005192.

[23] Pluess M, Conrad A, Wilhelm FH. Muscle tension in generalized anxiety disorder: a critical review of the literature. J Anxiety Disord. 2009;23:1-11.

[24] Manzoni GM, Pagnini F, Castelnuovo G, Molinari E. Relaxation training for anxiety: a ten-years systematic review with metaanalysis. BMC Psychiatry. 2008;8:41.

[25] Conrad A, Roth WT. Muscle relaxation therapy for anxiety disorders: it works but how? J Anxiety Disord. 2007;21: 243-264.

[26] Jorm AF, Christensen H, Griffiths KM, Parslow RA, Rodgers B, Blewitt KA. Effectiveness of complementary and self-help treatments for anxiety disorders. Med J Aust. 2004;181: 29-46.

[27] Jorm AF, Morgan AJ, Hetrick SE. Relaxation for depression. Cochrane Database Syst Rev. 2008:CD007142.

[28] Jorm AF, Christensen H, Griffiths KM, Rodgers B. Effectiveness of complementary and self-help treatments for depression. Med J Aust. 2002;176:84-96.

[29] Georgiev A, Probst M, De Hert M, Genova V, Tonkova A, Vancampfort D. Acute effects of progressive muscle relaxation on state anxiety and subjective well-being in chronic Bulgarian patients with schizophrenia. Psychiatr Danub. 2012; 24:367-372.

[30] Vancampfort D, Correll CU, Scheewe TW, Probst M, De Herdt A, Knapen J, et al. Progressive muscle relaxation in persons with schizophrenia: a systematic review of randomized controlled trials. Clin Rehabil. 2013;27:291-298.

[31] Dayapoğlu N, Tan M. Evaluation of the effect of progressive relaxation exercises on fatigue and sleep quality in patients with multiple sclerosis. J Altern Complement Med. 2012;18: 983-987.

[32] Zhao L, Wu H, Zhou X, Wang Q, Zhu W, Chen J. Effects of progressive muscular relaxation training on anxiety, depression and quality of life of endometriosis patients under gonadotrophin-releasing hormone agonist therapy. Eur $J$ Obstet Gynecol Reprod Biol. 2012;162:211-215.

[33] Bae BG, Oh SH, Park CO, Noh S, Noh JY, Kim KR, et al. Progressive muscle relaxation therapy for atopic dermatitis: objective assessment of efficacy. Acta Derm Venereol. 2012; 92:57-61.

[34] Sloman R. Relaxation and imagery for anxiety and depression control in community patients with advanced cancer. Cancer Nurs. 2002:25:432-435.

[35] Holland JC, Morrow GR, Schmale A, Derogatis L, Stefanek M, Berenson S, et al. A randomized clinical trial of alprazolam versus progressive muscle relaxation in cancer patients with anxiety and depressive symptoms. J Clin Oncol. 1991;9: 1004-1011.

[36] First MB, Spitzer RL, Gibbon M, Williams JBW. Structured Clinical Interview for DSM-IV-TR Axis I Disorders, Research Version, Patient Edition (SCID-I/P). New York: Biometrics Research, New York State Psychiatric Institute; 2002.

[37] Koch C. Fragebogen zur Abschätzung Psychosomatischen Krankheitsgeschehens (FAPK). Theoretische Grundlagen und Handanweisung (2. Auflage). Weinheim: Beltz Test GmbH; 1981.

[38] MacPherson H, Altman DG, Hammerschlag R, Li Y, Wu T, White A, et al. STRICTA Revision Group. Revised STandards for Reporting Interventions in Clinical Trials of Acupuncture 
(STRICTA): extending the CONSORT statement. Acupunct Med. 2010;28:83-93.

[39] Bernstein DA, Borkovec TD. Progressive relaxation training: a manual for the helping professions. Champaign, IL: Research Press; 1973.

[40] Hinkelmann K, Yassouridis A, Mass R, Tenge H, Kellner M, Jahn H, et al. CCK-4: Psychophysiological conditioning elicits features of spontaneous panic attacks. J Psychiatr Res. 2010; 44:1148-1153.

[41] Michalek-Sauberer A, Gusenleitner E, Gleiss A, Tepper G, Deusch E. Auricular acupuncture effectively reduces state anxiety before dental treatment-a randomized controlled trial. Clin Oral Investig. 2012;16:1517-1522.

[42] Karst M, Winterhalter M, Muente S, Francki B, Hondronikos A, Eckardt A, et al. Auricular acupuncture for dental anxiety: a randomized controlled trial. Anesth Analg. 2007;104: 295-300.

[43] Wu S, Liang J, Zhu X, Liu X, Miao D. Comparing the treatment effectiveness of body acupuncture and auricular acupuncture in preoperative anxiety treatment. J Res Med Sci. 2011;16:39-42.

[44] Wang SM, Punjala M, Weiss D, Anderson K, Kain ZN. Acupuncture as an adjunct for sedation during lithotripsy. $J$ Altern Complement Med. 2007;13:241-246.
[45] Wang SM, Kain ZN. Auricular acupuncture: a potential treatment for anxiety. Anesth Analg. 2001;92:548-553.

[46] Acar HV, Cuvaș O, Ceyhan A, Dikmen B. Acupuncture on Yintang point decreases preoperative anxiety. J Altern Complement Med. 2013;19:420-424.

[47] Paraskeva A, Melemeni A, Petropoulos G, Siafaka I, Fassoulaki A. Needling of the extra 1 point decreases BIS values and preoperative anxiety. Am J Chin Med. 2004;32: 789-794.

[48] Black S, Carey E, Webber A, Neish N, Gilbert R. Determining the efficacy of auricular acupuncture for reducing anxiety in patients withdrawing from psychoactive drugs. J Subst Abuse Treat. 2011;41:279-287.

[49] Fernandez E, Salem D, Swift JK, Ramtahal N. Meta-analysis of dropout from cognitive behavioral therapy: Magnitude, timing, and moderators. J Consult Clin Psychol. 2015;83: $1108-1122$.

[50] Mischoulon D, Brill CD, Ameral VE, Fava M, Yeung AS. A pilot study of acupuncture monotherapy in patients with major depressive disorder. J Affect Disord. 2012;141:469-473. 